\title{
Der geologische Bau pleistozäner Inlandgletschersedimente Norddeutschlands
}

\author{
F. GRUBE und TH. VOLLMER
}

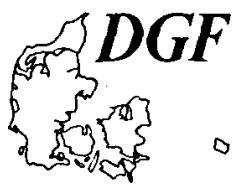

\begin{abstract}
Grube, F. \& Vollmer, Th.: Der geologische Bau pleistozäner Inlandgletschersedimente Norddeutschlands, Bull. geol. Soc. Denmark; vol. 34 pp. 13-25, Copenhagen, May, 30th, 1985.

https://doi.org/10.37570/bgsd-1985-34-02

The geologic structure and genetic aspects of inland galcier sediments are described on examples from northem Germany. Different types of structures are demonstrated ranging from slight undulation to intense subglacial folding and shearing. Inland glacier sediments are called all deposits laid down and/or de-formed by inland glaciers. These sediments resting on glacially undeformed basement include lodgement till (at top) and below of it shear till, melt-out and deformation till. A fundamental tool of modern till stratigraphy is the knowledge of the geologic structure more than are petrography and geochemistry of moraines at present.
\end{abstract}

F. Grube, Geologisches Landesamt Schleswig-Holstein, Mercatorstraße 7, D-2300 Kiel, BRD. Th. Vollmer, Geologisch-Paläontologisches Institut und Museum der Universität Hamburg, Bundesstraße 55, D-2000 Hamburg 13, BRD. July 24th, 1984.

\section{Einleitung}

Gletscher nehmen bei ihrer Bewegung Gesteinsmaterial auf, transportieren diesen Schutt unter gleichzeitiger Aufbereitung und bilden daraus Grundmoränen. Durch sub- bis proglaziale Deformation werden die Sedimente der Gletscherbasis zu Sohlmoränen (Scher-, Stauch- und Schmelzmoränen) umgeformt. Alle aus dem Indlandeis abgelagerten und durch Gletscherauflast und -schub deformierten Gesteine werden als Gletschersedimente oder Moränen (Material: Till) bezeichnet. In einem Idealprofil läßt sich folgende Sequenz beobachten (vereinfachtes Schema):

$\leftarrow$ Gletscherfließrichtung

Inlandeis

Gehalt an Lokalmaterial $\leftarrow$ zunehmend $\downarrow$

Endmoräne Grundmoräne
$\begin{aligned} & \text { Stauchmoräne, Sohlmoräne } \\ & \text { proglazial }\end{aligned}$

Nicht deformierter Untergrund

Moränen entstehen unter dem Einfluß verschiedener teils dominanter, teils steuernder Faktoren, die miteinander wechlselwirken können:
Inlandeis:

Mächtigkeit (Sohldruck)

Geschiebeführung

Temperatur

Fließgeschwindigkeit

Bewegungsart (plastisch fließend; starr gleitend)

Untergrund:

Relief, Gefälle (Größe und Richtung)

Bodenart(en), bodenmechanische Kennwerte

Wassergehalt, Porenwasserdruck

Temperatur (gefrorener/nicht gefrorener Zustand; bruchlose Faltung durch Regelation).

Erhöhung des Sohldruckes z.B. wirkt gefrierpunktsniedrigend, so da $\beta$ ein Inlandgletscher genügender Mächtigkeit plastisch auf partiell nicht gefrorenem Untergrund fließen kann. Dabei kann sich die Deformation des Untergrundes auf Zerscherung einer geringmächtigen Gesteinspartie beschränken (Schermoräne). Bei geringerer Eismächtigkeit kann der Gletscher festfrieren und teilweise als Toteis ortsfest werden oder durch weitere Eigenbewegung bzw. Andrängen proximaler Gletscherteile Stauchungen von groBem Tiefgang verursachen. Diese können bei genügenden Kraftschlu $ß$ durch tiefreichende $\mathrm{Ge}$ frornis Schuppenstrukturen sowie in geeigneten 


\begin{tabular}{|c|c|c|c|c|}
\hline & \multirow{2}{*}{$\begin{array}{l}\text { Grundmoränen (Fernmoränen) } \\
\text { (Geschiebemergel, Grundtill) }\end{array}$} & \multicolumn{3}{|c|}{ Sohlmoränen (Sohltill) } \\
\hline & & $\begin{array}{l}\text { Schermoränen } \\
\text { (Schertill) }\end{array}$ & $\begin{array}{l}\text { Stauchmoränen } \\
\text { (Stauchtill) }\end{array}$ & $\begin{array}{l}\text { Schmelzmoränen } \\
\text { (Schmelztill) }\end{array}$ \\
\hline Petrographie & $\begin{array}{l}\text { sandig-tonige Matrix mit } \\
\text { "unverdauten" Steinen, } \\
\text { Blöcken, Schollen, Sor- } \\
\text { tierung meist gering; } \\
\text { skandinavisches Material } \\
\text { vorwiegend }\end{array}$ & $\begin{array}{l}\text { Geschiebe- } \\
\text { mergel, Lokal- } \\
\text { material wie } \\
\text { z.B älterer } \\
\text { Till, Glazi- } \\
\text { fluvium, Prä- } \\
\text { pleistozän }\end{array}$ & $\begin{array}{l}\text { Lokalmaterial } \\
\text { wie z.B.Glazi- } \\
\text { fluvium, } \\
\text { Interglazial- } \\
\text { tone; wechseln- } \\
\text { de Anteile } \\
\text { synchroner und } \\
\text { älterer Ge- } \\
\text { schiebemergel }\end{array}$ & $\begin{array}{l}\text { geschiebe- } \\
\text { mergelähnlich, } \\
\text { gröber und } \\
\text { sandiger als } \\
\text { syngentische } \\
\text { Grundmoräne }\end{array}$ \\
\hline $\begin{array}{l}\text { Relative Fazies- } \\
\text { beständigkeit }\end{array}$ & & & & \\
\hline $\begin{array}{ll}\text { a) } & \text { radial } \\
\text { b) } & \text { tangential } \\
\text { c) } & \text { vertikal }\end{array}$ & $\begin{array}{l}\text { meist hoch, } \\
\mathrm{a}>\mathrm{b} \geqq \mathrm{c}\end{array}$ & $\begin{array}{l}\text { gering, } \\
\mathrm{a}, \mathrm{b}>\mathrm{c}\end{array}$ & $\begin{array}{l}\text { gering, } \\
\mathrm{a}<\mathrm{b} \gtrless \mathrm{c}\end{array}$ & $\begin{array}{l}\text { ähnlich Grund- } \\
\text { moränen }\end{array}$ \\
\hline $\begin{array}{l}\text { Quotient } \\
\text { Fern-/Lokalmaterial }\end{array}$ & meist $\gg 1$ & $\begin{array}{l}\text { wechselnd, } \\
\text { oft } \gtrless 1\end{array}$ & $\begin{array}{l}\text { wechselnd, be- } \\
\text { vorzugt }<1\end{array}$ & $\begin{array}{l}\text { wechselnd, } \\
\text { oft }>1\end{array}$ \\
\hline $\begin{array}{l}\text { Transportweite von } \\
50 \% \text { des Materials }\end{array}$ & $>100 \mathrm{~km}$ & $\begin{array}{l}\text { Millimeter- } \\
\text { bis? Deka- } \\
\text { meterbereich }\end{array}$ & $\begin{array}{l}\text { Dezimeter- bis } \\
\text { Kilometer- } \\
\text { bereich }\end{array}$ & $\begin{array}{l}\text { Kilometerbe- } \\
\text { reich und } \\
\text { größer }\end{array}$ \\
\hline Mächtigkeit & Meterbereich bis $>80 \mathrm{~m}$ & $\begin{array}{l}\text { Dezimeter- } \\
\text { bis Meterbe- } \\
\text { reich }\end{array}$ & $\begin{array}{l}\text { (Meter-) Deka- } \\
\text { meterbereich, } \\
\text { bis }>100 \mathrm{~m}\end{array}$ & $\begin{array}{l}\text { Dezimeter } \\
\text { (-Meter) } \\
\text { bereich }\end{array}$ \\
\hline $\begin{array}{l}\text { durchschnittlicher } \\
\text { Quotient Fläche/ } \\
\text { Mächtigkeit }\end{array}$ & sehr hoch & $\begin{array}{l}\text { wechselnd, } \\
\text { meist hoch }\end{array}$ & meist niedrig & meist hoch \\
\hline $\begin{array}{l}\text { bestimmende Gefüge- } \\
\text { elemente }\end{array}$ & $\begin{array}{l}\text { Schubklïfte, subhori- } \\
\text { zontale Scherflächen }\end{array}$ & $\begin{array}{l}\text { subhorizon- } \\
\text { tale Scher- } \\
\text { flächen }\end{array}$ & $\begin{array}{l}\text { steile(-flache) } \\
\text { Scherflächen, } \\
\text { Schuppen, Falten, } \\
\text { irreguläre For- } \\
\text { men }\end{array}$ & $\begin{array}{l}\text { z.T. wie } \\
\text { Grundmoräne, } \\
\text { weniger aus- } \\
\text { prägt }\end{array}$ \\
\hline $\begin{array}{l}\text { Form; } \\
\text { Sedimentation }\end{array}$ & deckenartig, flächenhaft & $\begin{array}{l}\text { schichtförmig; } \\
\text { flächenhaft }\end{array}$ & $\begin{array}{l}\text { oft gestreckt; } \\
\text { bevorzugt } \\
\text { linear }\end{array}$ & $\begin{array}{l}\text { schichtförmig, } \\
\text { gangförmig, } \\
\text { flächenhaft }\end{array}$ \\
\hline glaziale Fazies & $\begin{array}{l}\text { inglazial; an der } \\
\text { Gletscherbasis }\end{array}$ & $\begin{array}{l}\text { subglazial; } \\
\text { Basis Grund- } \\
\text { moräne bis } \\
\text { tiefste Sohl- } \\
\text { moräne }\end{array}$ & $\begin{array}{l}\text { subglazial; } \\
\text { proximal bis } \\
\text { frontnah } \\
\text { (=proglazial) }\end{array}$ & $\begin{array}{l}\text { subglazial; } \\
\text { Basis Grund- } \\
\text { moräne bis } \\
\text { tiefste Sohl- } \\
\text { moräne }\end{array}$ \\
\hline
\end{tabular}

Tab. 1. Übersicht der Merkmale von Inlandgletschersedimenten (vereinfachtes Schema).

Table 1. Some important properties of tills.

Bodenarten bevorzugt Faltenkomplexe erzeugen.

Ein Einfluß des Untergrundes zeigt sich z.B. daran, daB Grundmoränen in präexistenten glazifluvialen oder vom Gletscher selbst exarierten Senken (Glazielle, Gripp 1975) gewöhnlich mächtiger entwickelt sind als auf Erhebungen des
Untergrundes. Die Flanken subglazialer Hohlformen können ferner Anlaß zu Eisstau und tiefgründiger Stauchung geben (Wilke 1982). In diesem Sinne kann auch, äquivalent einer distalen Rinnenflanke, bewegungslos gewordenes Eis (Toteis) als Widerlager bei der Bildung von Stauchkomplexen wirken. Durch weitere Wech- 
selwirkungen zwischen Gletscher und Untergrund entstehen die Vollformen der Grundmoränenlandschaft:

"Felsdrumlins« (Kern aus älterem Material), Drumlins (überwiegend syngenetischer Geschiebemergel),

Rogenmoränen (wie Drumlins, quer zur Eisfließrichtung orientiert).

\section{Petrographie der Moränen}

Die Petrographie wird zugunsten des geologischen Baues der Moränen hier nur kurz abgehandelt (s. auch Tab. 1). Der Geschiebemergel einer Grundmoräne ist ein relativ homogenes, feinkornreiches Lockergestein, das vorwiegend aus skandinavischem Material besteht und über größere Distanzen ähnliche Zusammensetzung bzw. allmähliche, fließende Faziesübergänge aufweist. Sohlmoränen dagegen sind allenfalls in kleineren Teilbereichen (Schollen, Schuppen) gleichartig, im allgemeinen aber heterogen zusammengesetzt. Sie können aus den verschiedensten Gesteinen wie glazifluvialen Sedimenten, interglazialen Meerestonen, Tertiärton, Schreibkreide, Geschiebemergel älterer Vergletscherungen u.a. bestehen; oft überwiegen Lokalgesteine. Fazieswechsel innerhalb einer Sohlmoräne sind kleinräumig und meist sprunghaft.

\section{Geologischer Bau von Moränen}

Die einzelnen Moränentypen lassen z.T. gemeinsame Baumerkmale erkennen, die in unterschiedlichem $\mathrm{Ma} ß$ entwickelt sind: Regelung von Einzelkörnern bis zu Schollen und sonstige Anisotropien wie Plättung und B-axiale Gefüge. Die räumliche Orientierung der Gefügeelemente ist von der Bewegungsrichtung des Inlandeises und dem Zusammenwirken von glazialer Dynamik und Einflüssen des Untergrundes abhängig. Die Unterschiede im Bau der Moränen ergeben sich aus jeweils vorherrschendem oder ausschließli-

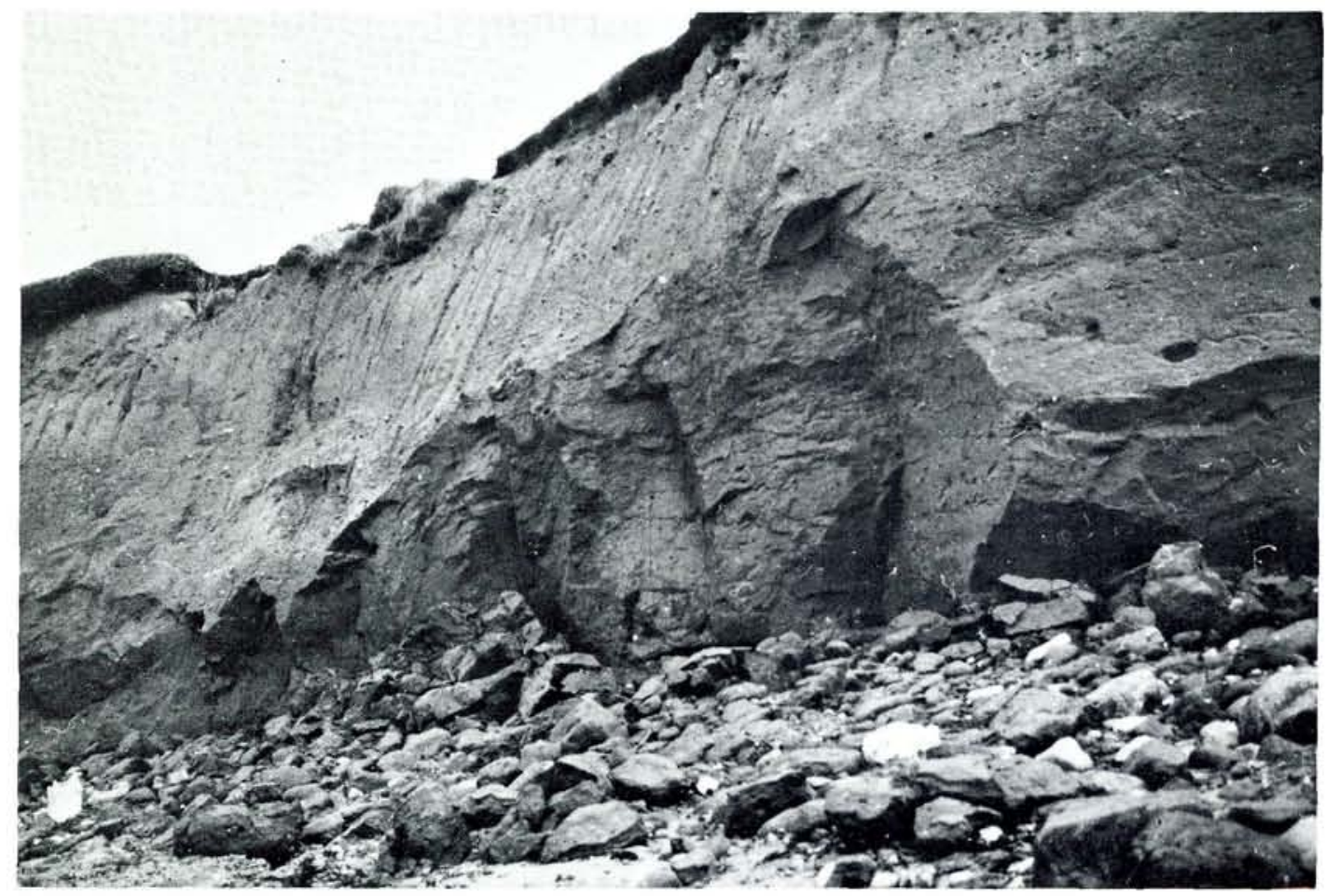

Foto I. Mohrsche Klüftung in einer Moräne, Brandungszone am Emmerlev-Kliff, phot. Dipl. Geol. BOLL.

Photo 1. Moraine showing Mohr shears, surf zone at Emmerlev-Kliff, phot. Dipl. Geol. BOLL. 


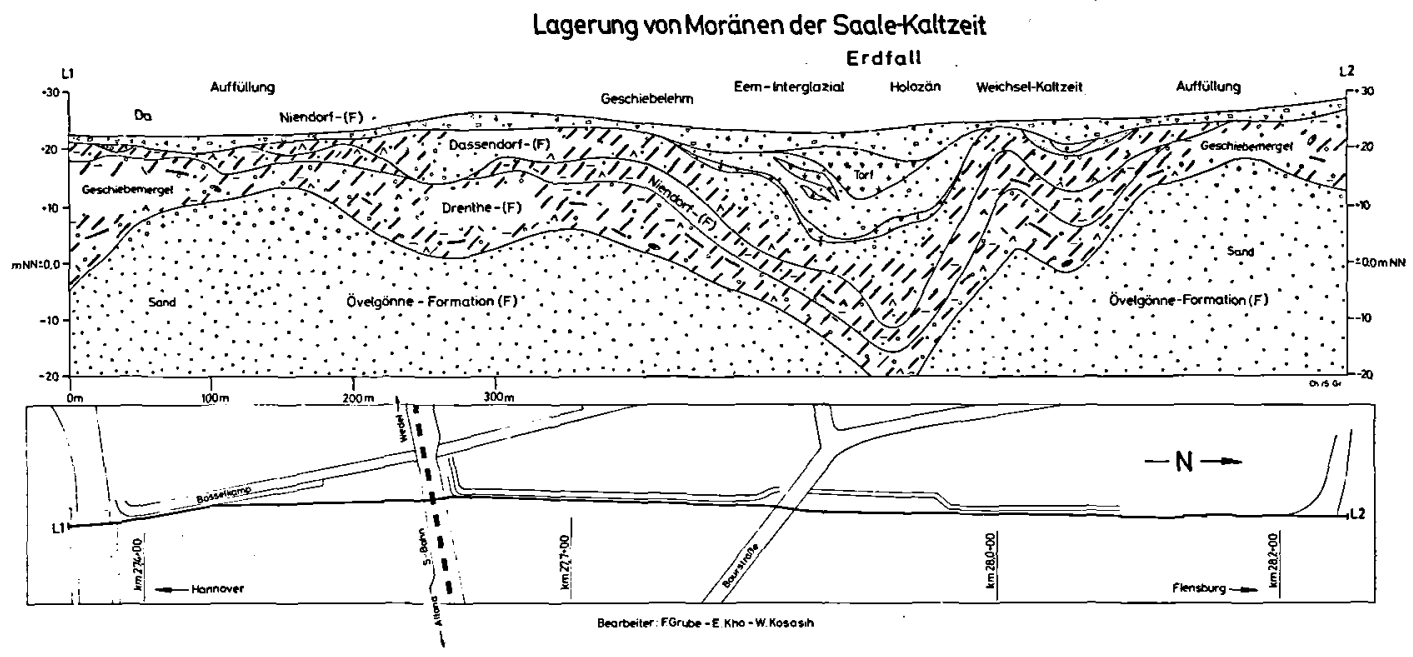

Abb. 1. Flachwellige Lagerung dreier Grundmoränen der Saale-Kaltzeit und sekundäre Lagerungsstörungen in einer Karstregion; Straßeneinschnitt in Hamburg-Bahrenfeld.

chem Vorkommen bestimmter Baumerkmale. In manchen Moränen sind außer syngenetischen Gefügeelementen auch ältere, tektonische oder sedimentäre Gefüge erhalten.

\section{Grundmoränen}

Die einzelnen durch ihre petrographische $\mathrm{Zu}$ sammensetzung und geotechnische Eigenschaften unterschiedenen Grundmoränen zeigen Gemeinsamkeiten des Baues: Flachwellige Lagerung, Mohrsche Kluftflächen und andere, im unverwitterten Till nicht immer deutlich sichtbare Klüfte sowie gelegentlich subhorizontale Scherflächen. Die schon von Richter (1929: 43-44; 1930: 82-83) erkannte Zerklüftung ist wahrscheinlich ein primäres Charakteristikum der Grundmoränen, das durch Gletscherschub entsteht und nach Richter die letzte Bewegungsphase des Eises dokumentiert. Zur Klärung dieses Sachverhaltes bedarf es weiterer Untersuchungen (Mickelson, Waldermarson \& Johansson 1981), zumal die Klüftung von Grundmoränen auch praktische Bedeutung hat (Standsicherheit, Tragfähigkeit): So verursachte die Klüftung weichselzeitlichen Tills beim Bau des Fährhafens Puttgarden/Fehmarn technische Schwierigkeiten. An Geschiebemergel-Steilufern (s. Foto 1) begünstigt die Klüftung sturmflutbe-
Fig. 1. Slight undulation of three saalian base moraines and secondary dislocation in karst region; road-cut in Hamburg-Bahrenfeld.

dingte Massenbewegungen (Lösen und Abgleiten von Kluftkörpern). Die Mächtigkeit von Grundmoränen kann auf engem Raum von wenigen Metern bis zu $>30 \mathrm{~m}$ wechseln, örtlich sogar $>80$ $\mathrm{m}$ betragen. Diese Erscheinung wie auch die flachwellige Lagerung wird am Beispiel einer saalezeitlichen Schichtfolge deutlich, die durch den Autobahnbau in Hamburg-Bahrenfeld aufgeschlossen war (s. Abb. 1).

\section{Grundmoränen, Hamburg-Bahrenfeld}

Im Hangenden der sandigen fluvialen Övelgönne-Formation folgen drei Grundmoränen aufeinander: a) schluffreicher sandiger Till der Drenthe-Formation (ältere Saalevergletscherung), b) toniger, kreide- und flintreicher Till der Niendorf-Formation, c) inhomogener, oft sandiger Till der Dassendorf-Formation (jüngerer Saale-Vergletscherung). Zugehörige glazifluviale und interstadiale Bildungen fehlen weitgehend, nur im nördlichen Profilteil sind glazifluviale Sande der jüngeren Dassendorf-Formation erhalten geblieben. Das Hangende bilden in einem Erdfallbereich vor der Abtragung geschützte Eem- und Holozäntorfe sowie weichselzeitliche periglaziale und niveofluviale Sedimente. Die drei Saalemoränen sind schichtförmige Körper mit subparallelen Grenzen und flachwelligem 
Großbau (Abb. 1 ist vierfach überhöht!); sie stehen großenteils in parakonkordantem, örtlich in schwach winkeldiskordantem Verband miteinander. Der wellige Großbau ist durch Wellenlängen von rund $100 \mathrm{~m}$ bis $200 \mathrm{~m}$ und Amplituden von 5 $\mathrm{m}$ bis $20 \mathrm{~m}$ charakterisiert. Ihm sind jüngere Störungen (s. unten) Im Bereich von Erdfällen im nördlichen Profilabschnitt aufgeprägt.

Die Drenthe-Moräne durchzieht den gesamten Aufschluß als geschlossene Schicht von $5 \mathrm{~m}$ bis 15 $\mathrm{m}$, im Westen $>20 \mathrm{~m}$ Mächtigkeit. Niendorf- und Dassendorf-Formation sind maximal $5 \mathrm{~m}$ bzw. 10 $m$ mächtig. Durch den Vorstoß des jüngsten Saale-Gletschers ist die Niendorf-Moräne örtlich abgetragen, so daß hier Drenthe-Till direkt von Dassendorf-Formation überlagert wird. Auch diese liegt wegen jüngerer Abtragung nicht mehr als vollständige Decke vor. Die Mächtigkeitsunterschiede gehen nicht allein auf Erosion und Exaration, sondern teilweise auf das ehemalige subglaziale Relief zurück. So steigt die Mächtigkeit der Drenthe-Formation von $<5 \mathrm{~m}$ auf einer Hochlage im südlichen Profilteil auf $>20 \mathrm{~m}$ in einer südlich anschließenden Depression.

Außer Erosions- und Exarationserscheinungen sind keine wesentlichen Lagerungsstörungen der älteren Moränen durch jüngere Eisvorstöße festzustellen.

\section{Sohlmoränen}

Der Begriff Sohlmoräne bezeichnet einen von Verschiebungsflächen begrenzten und durchsetzten heterogenen Gesteinskörper im Liegenden der Grundmoräne. Er umfaßt alle subglazial durch Gletscherschub und -auflast transportierten und deformierten Gesteine von der Basis der Grundmoräne bis zur Oberfläche des nicht bewegten Untergrundes. Zur hangenden Grundmoräne besteht meist ein fließender Übergang, gegen die Basis in der Regel eine scharfe Grenzfläche. Die Gesteine oberhalb dieser Grenzfläche sind aus ihrem ursprünglichen Verband gelöst, zerschert, verfaltet, zerbrochen, zerklüftet und (unterschiedlich weit) vom Ursprungsort verlagert worden. Unterhalb der Basis sind, abgesehen von einzelnen Spalten und Klüften gewöhnlich keine Dislokationen festzustellen. Selten finden sich mit Schmelztill gefüllte Spalten geringer Öffnungsweite, die von der Basis einer Sohlmo- räne schräg in den Untergrund setzen und nach wenigen Metern auskeilen (z.B. Rotes Kliff/Sylt Winter 82/83). Sie dokumentieren die im Frühstadium steckengebliebene Abspanung einer größtenteils noch im Verband sitzenden Schuppe des Untergrundes. Nur in wenigen Fällen läßt sich die tiefste glaziale Bewegungsfläche mit erheblichen Schwierigkeiten definieren, und zwar bei einer beginnenden Faltung oder dem Anfangsstadium der Verschuppung des Untergrundes. Auch in den »Felskernen « der Drumlins sind Aufpressungen beobachtet worden, die noch nicht vom Horizontalschub des Gletschers erfaßt wurden.

Grenzflächen und interne Scherflächen einer Sohlmoräne sind häufig Materialgrenzen; sie können aber auch einheitliche Gesteine, z.B. Sande durchsclagen und sind dann nur unter günstigen Tagesaufschlußverhältnissen nachweisbar (s. unten).

Die Abgrenzung einer Sohlmoräne mittels gewöhnlicher Erkundungsbohrungen wie auch in der herkömmlichen Quartärkartierung ist in solchen Fällen schwierig oder undurchführbar (Bedeutung der Aufsclußgröße). Trotzdem ist eine Ausgliederung der Sohlmoräne als glaziale Fazies, insbesondere die Abgrenzung gegen den autochthonen Untergrund, nicht nur wissenschaftlich, sondern auch praktisch sinnvoll; dies bestätigen Beispiele aus der Ingenieurgeologie, $\mathrm{Hy}$ drogeologie und Lagerstättenkunde. So kan es z.B. für einen unter künstlicher Grundwasserhaltung arbeitenden Tongewinnungsbetrieb zur Existenzfrage werden, ob das in Abbau stehenden Tonvorkommen autochthon ist oder, umgeben von wassererfüllten Sanden, als Scholle in einer Sohlmoräne »schwimmt«.

Eine Sohlmoräne läßt sich in drei Subfazies gliedern, die in unterschiedlichen Anteilen vertreten sein können. Vertikale und laterale Verbreitung der einzelnen Subfazies und deres geologischer Bau ermöglichen Rückschlüsse auf Transport- und Deformationsart und Relativgeschwindigkeit des Materieltransports. Entsprechend können näherungsbeweise Profile der Relativgeschwindigkeit des glazitektonischen Transports abgeleitet werden. Den Sohlmoränen sind auch die von Carlé $(1939,1939 a)$ untersuchten und erstmals detailliert beschriebenden Moränenstrukturen zuzuordnen. Hervorragende moderne tektonische Analysen finden sich vor allem 
in der dänischen Literatur. Berthelsen (1981) führte aus dem Archaikum bewährte tektonische Arbeitsmethoden in die Quartärtektonik des Pleistozän ein. Auf einem INQUA-Symposium auf Moen wurden von M. Houmark-Nielsen, E. M. Jacobsen, P. E. Nielsen, K. S. Petersen, S. Sjørring u.a. neue Dokumentationen dänischer Kliff-Aufschlüsse mit tektonischen Analysen und Synthesen vorgetragen. ter Wee (1962), van der Wateren (1981), Ruegg \& Zandstra (1981) und Rappol (1983) vertieften die Kenntnisse über den Aufbau der Stauchmoränen der Niederlande. Ruszczyńskaszenajch (1981) faßte die umfangreichen glazialtektonischen Forschungen in Polen zusammen. Prange (1975) nutzte die vorzüglichen Kliff-Aufschlüsse der Ostseeküste Schleswig-Holsteins zu intensiven gefügekundlichen Untersuchungen weichselzeitlicher Moränen.

\section{Schermoränen}

Schermoränen finden sich in unterschiedlichen Positionen innerhalb einer Sohlmoräne, anscheinend aber bevorzugt an der Basis der Grundmoräne. Sie können eine Sohlmoräne auch allein vertreten. Schermoränen sind vor allem durch Plättung und engständige Scherung parallel zur ab-Ebene gekennzeichnet. Daraus resultiert oft eine subhorizontale Pseudoschichtung von Geschiebemergel- und dünnen (Millimeter bis Zentimeter) Sand- und Tonlagen oder anderem Material.

Moränen dieser Art beschrieb schon Richter (1929: 36ff.). Er deutete die subhorizontalen Lagen als Scharen von Abscherungsflächen. Bei hochgradiger Zerscherung treten flachliegende, oft gegen die Eisfließrichtung einfallende Verschiebungsflächen so zahlreich und engständig auf, daß mit Richter (1930: 81) von einer "Gleitschieferung« gesprochen werden kann. Diese "geschieferte« Zone zieht sich auch durch ältere überfahrene Sande, Silte usw. hindurch: In einem Tagesaufscluß des U-Bahn-Neubaues in Hamburg-Niendorf ließ sich ein ca. 0,3 m mächtiger Schertill von der Sohle der jüngsten SaaleGrundmoräne durch eine drumlinartige Sandaufragung verfolgen, auf der die Moräne selbst abgetragen war. In einem ständig zugänglichen natürlichen Aufschluß am Prallhang der Elbe bei Kuhgrund/Lauenburg kann ein Schertill in der
Sohlmoräne der Niendorf-Formation beobachtet werden.

Die geringe Mächtigkeit und der gleichförmige "papierstapelartige« Aufbau aus zahlreichen dünnen Gleitbrettern charakterisieren Schermoränen als Zonen hoher vertikaler Gescheindigkeitsgradienten mit relativ homogenem oder quasikontinuierlichem Geschwindigkeitsprofil.

\section{Schmelzmoränen}

Schmelzmoränen treten vorzugsweise im tiefsten Teil der Sohlmoräne, untergeordnet im Liegenden der Grundmoräne auf. Sie bestehen aus tonig-siltigem Sand (Sandtill), in dem Langachsen der Geschiebe oft eine klare Regelung parallel zur EisflieBrichtung aufweisen. Diese schon von Richter (1929: 40-41) beschriebenen, auch in Spalten erindringende Moränen sind gröberkörnig und stärker sandig als die zugehörigen Grundmoränen. Der Terminus Schmelzmoränen wurde auf der INQUA 1982 in Moskau von H. J. Stephan vorgeschlagen.

\section{Stauchmoränen}

Stauchmoränen bestehen aus Schuppen und Schollen von $<1 \mathrm{~m}^{3}$ bis zu $\gg 10^{6} \mathrm{~m}^{3} \mathrm{Größe}$ und mannigfaltigen Formen: annähernd kugelig, ellipsoidisch, planar, gebogen, konkav-konvex, fetzenartig usw. Als Schuppen werden hier vergleichsweise geringfügig transportierte, bevorzugt reguläre Gesteinskörper verstanden, die von großenteils steilen Scherflächen begrenzt und durch diese von anderen Schuppen odèr dem Untergrund abgesetzt sind. Entlang der Schuppengrenzen hat keine wesentliche Materialdurchmischung stattgefunden. Schollen stecken isoliert in glazitektonisch durchmischten Gesteinen wie Geschiebemergel oder vergleichbaren polymikten Sedimenten. Sie sind meist weiter transportiert als Schuppen und stellen diesen gegenüber ein fortgeschrittenes Stadium der Einarbeitung von Gesteinsmaterial in eine Moräne dar. Die obige Definition hat vorläufigen Charakter, sie ist in langjährigen Geländebeobachtungen gewachsen. Das Gefüge von Stauchmoränen zeigt gewöhnlich glazitektonische Regelung mit deutlichen Beziehungen zur Gletscherfließrichtung: dach- 
ziegelartige Lagerung »stromaufwärts« einfallender Schuppen; Anordnung von Scherflächen, Langachsen und Faltenachsen häufig quer zur Fließrichtung; in der Aufsicht sichelförmige Schuppen, deren konvexe oder konkave Seite in die Eisfließrichtung weist; Einfallen von s-Flächen und Achsenflächen bevorzugt gegen die Eisfließrichtung. Stephan (1981) beobachtete parallel zur Gletscherfließrichtung streichende Deformationen. Daneben gibt es chaotische Lagerungsformen ohne deutlich erkennbare tektonische Hauptrichtungen und, als anderes Extrem, wenige Meter mächtige subhorizontale Schollen von mehreren 100 Metern Ausdehnung (z.B. Elster-Schollen in Drenthe-Till, zeitweilig aufgeschlossen, Baugrube Elbe-Seitenkanal b. Uelzen/Niedersachsen). Der Innenbau der Schuppen und Schollen weist syngenetische Bewegungsflächen und meist in Eisfließrichtung vergente Falten auf, die in unterschiedlichen Anteilen vertreten und/oder zu Schuppenfalten usw. kombiniert sein können. Trotz der glazitektonischen Beanspruchung (Transportweite u.a.) gibt es Schollen, deren Primärgefüge durch syntektonische Frostverfestigung erhalten blieb und intern nicht oder nur wenig deformiert worden ist. So sind Schräg- oder Warvenschichten in einzelnen Schollen vorzüglich erhalten. An der Sohle einer jungen Saale-Moräne wurde am Hamburger Hauptbahnhof eine komplette Scholle von Meerestonen des Holstein-Interglazials beobachtet. Das nur schwach geneigte Vorkommen, bestehend aus Lauenburger Tonen, holsteinzeitlicher Süßwassermudde und Cardiensanden von Hamburg-Hummelsbüttel lieferte das Material für litho- und biostratigraphische Arbeiten (Valeton 1980, Knudsen 1980). Mit Hilfe gut erhaltener Primärgefüge, insbesondere Schrägschichtung, ist es möglich, (Extern-)Rotationen nachzuweisen und deren Mindestbeträge abzuschätzen.

Nach ihrem geologischen Bau können Stauchmoränen als Bereiche mit inhomogener Geschwindigkeitsverteilung des glazitektonischen Transports gedeutet werden. Aus den Aufschlußaufnahmen und Messungen (s. unten) läßt sich eine Anzahl möglicher Prozesse ableiten, die bei der Entstehung von Schuppen usw. sowie der intern Gefüge wirken; zwei anscheinend bevorzugte Abläufe sind:
A 1) Anlage konkaver Scherflächen im Untergrund und

2) grundbruchartige Aufpressung von Schuppen unter Einkippung gegen die Eisfließrichtung,

3) Entwicklung der Schuppen zu Phacoiden und Schollen unter Ausdünnung bzw. Anstau und Rotation.

$B$ 1) Abspanung (z.T. wohl durch Fließen begünstigt) von Schuppen aus dem Untergrund,

2) Aufrichtung in der Eisfließrichtung, Steilstellung,

3) Überkippung,

4) Auswalzung oder Ablösung und weitere Entwicklung wie unter $A$ 3).

Während der Aufrichtung von Untergrundpartien durch den Prozeß $B$ kann anfangs offenbar plastisches Fließen vorherrschen und primär Falten erzeugen, die erst in einem späteren Stadium durch Bildung definierter Abscherungsflächen zu eigentlichen Schuppen werden.

Außer den hier nicht behandelten Stirnstauchmoränen werden zwei Typen subglazialer Stauchmoränen unterschieden (Blickrichtung vom Eiszentrum zum Eisrand):

a) Subglazial-proximale Stauchmoränen fern der Gletscherfront, induziert durch Wechselwirkung Gletscher/Untergrund oder proximale Toteismassen, morphologisch meist weniger deutlich als Rücken ausgeprägt als b).

b) Subglazial-distale (frontnahe) Stauchmoränen (Stauchwälle)

nahe der Gletscherfront, induziert durch Toteis der Gletscherstirn (Widerlager), morphologisches Höhenwachstum und Relief stärker ausgeprägt als bei a).

\section{Beispiele von Stauchmoränen}

Der Schübarg/Hoisbüttel (Grube 1972) und die Hüttener Berge (Gripp 1964) sind auffallende Höhenzüge in der Randregion der Weichselvereisung. Saalezeitliche Stauchmoränen sind aus dem Niederrheingebiet (z.B. Braun 1964) und aus Niedersachsen (z.B. Lang 1963) beschrieben und gehören zu den kompliziertesten Strukturen 
der Flachlandkartierung (K. D. Meyer; H. C. Höfle). Nördlich der Elbe zählen das Höhengebiet von Wacken/Itzehoe, der Ketelvierthberg bei Neumünster und die Blankeneser Berge/ Hamburg (Wilke 1982) zu den bekanntesten Moränenwällen der Saale-Kaltzeit. Morphologie und innere Bau von Stauchzonen wurden u.a. bei den Wartenbergen/Ahrensburg (Grube \& Homci 1975) und dem »Staumoränenzug "Warsower Plateau - Brunn - Buchheide in Pommern (Richter 1929a) miteinander verglichen. Einzelstrukturen analysierte T. Vollmer (1982) am Ketelvierthberg/Neumünster und in Muldsberg bei Itzehoe. Einige in jüngerer Zeit untersuchte Beispiele von Stauchmoränen sind nachstehend erläutert.

\section{Subglazial-proximale Stauchmoränen}

\section{Beispiel Hamburg}

In Hamburg-Hohenfelde wurde der Übergang einer ungestörten Schichtfolge in Stauchtill in ausgedehnten Verkehrstunnelaufschlüssen kartiert (Grube 1967, Tafel 40). Die ungestörte Folge besteht aus saalezeitlichen Schichten, vom Liegenden zum Hangenden: Drenthe-Grundmoräne, Drenthe-Nachschüttsande und -kiese, Flußsande mit Moosresten (Wandsbeker Interstadial), Niendorf-Vorschüttsande, Niendorf-Grundmoräne. Der Übergang zu der östlich gelegenen Stauchzone wird durch Mächtigkeitsreduktion der Sandhorizonte eingeleitet. Die Stauchzone beginnt mit einfachem Faltenbau der gesamten Serie, der nach Osten zunehmend von Überschiebungen bestimmt wird. Östlich dieser wenige 100 Meter breiten Übergangszone schließt sich ein über viele Kilometer anhaltender komplizierter Schuppen- und Faltenbau an. Die Stauchzone hat keine besonderen morphologischen Merkmale. Ihr geologischer Bau wird genetisch als Grenzbereich zwischen einem westlich gelegenen Toteisgebiet mit ungestörten Liegendschichten und einem von Osten andrängenden Gletscher gedeutet.

In Hamburg-Altona war durch Verkehrstunnelbau ein vom jüngsten Saale-Gletscher aufgepreßter und überfahrener ehemaliger »Felsdrumlin« aus saalezeitlichen Schichten aufgeschlossen. Ein wesentlicher glazitektonischer Transport war

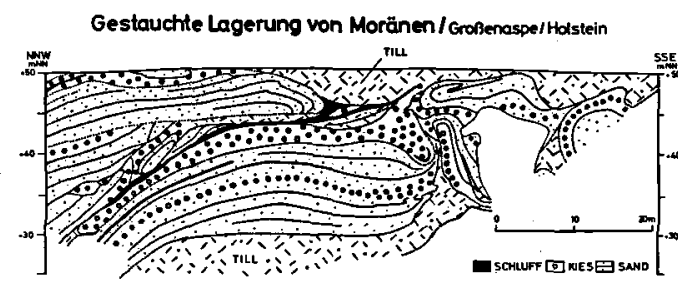

Abb. 2. Faltung und Schuppung glazigener, glazifluvialer und glazilakustriner Sedimente ein einem Stauchwall; Sandgrube Großenaspe/Neumünster, östlicher Abbaustoß, Darstellung als Saigerriß; geol. Aufnahme: T. Vollmer, 1982; Originalmaßstab $1: 100$.

Fig. 2. Folding and imbrication of glaciogenic, glaciofluvial and glaciolacustrine deposits in ice-pushed ridge; Sand pit Großenaspe/Neumünster, eastern slope, shown as vertical section; $T$. Vollmer, 1982; original scale was 1:100.

nicht festzustellen. Im Innern des Drumlins finden sich weit aushaltende Partien scheinbar ungestörter, flachlagernder Schichten, in Abständen von mehreren 100 Metern unvermittelt durch Schuppen- und Faltenzonen gegeneinander versetzt. Ähnliches erkannte Köster (1957: 570) am Wulfener Berg.

\section{Beispiel Muldsberg}

In der Tongrube Muldsberg nördlich Itzehoe/ Westholstein werden Meerestone der ausgehenden Elster-Kaltzeit und der Holstein-Warmzeit abgebaut. Der bis zu $40 \mathrm{~m}$ tiefe Abbau (unterste Sohle auf ca. $-5 \mathrm{~m} \mathrm{NN}$ ) hat, vom Liegenden zum Hangenden, folgendes Profil erschlossen: fetter bis stark sandiger schwarzer Ton (Elster); feinschichtige braungraue Holstein-Tone mit hellen Siltlagen sowie einzelnen faustgroßen Steinen und Grobsandlinsen, die wahrscheinlich durch winterliches Treibeis in einer Förde verfrachtet wurden; rote Tone (nach Khoo 1972, sekundär rotgefärbt); fossilreiche hellgraue Muscheltone (Menke 1982); bis zu > $10 \mathrm{~m}$ meist feinkörnige Sande (Holstein) mit Torf; örtliche dunkelgraue sandige Geschiebemergel (Saale). Die Tone sind mindestens mehrere Dekameter, möglicherweise $>100 \mathrm{~m}$ mächtig; genaue Werte lassen sich wegen der komplizierten Lagerung nicht angeben.

Die geologische Aufnahme des nördlichen Abbaustoßes (Abb. 3) zeigt eine starke westvergente Verfaltung und teilweise (kartierbare) Zerscherung der Tone. Amplituden und Spannweiten der Falten liegen in Zentimeter- bis Dekame- 
terbereich. Der größere Teil der Faltenachsen streicht bei insgesamt weiter Streuung der Raumlagen ungefähr NNW/SSE bis NW/SE, wobei Abtauchwinkel von $5^{\circ}$ bis $20^{\circ}$ vorherrshen. Die weniger häufig vertretenen N/S bis NE/SW streichenden Achsen tendieren stärker zu steilerem Abtauchen. Infolge der Westvergenz ist vorwiegend östliches, teilweise überkipptes Schichteinfallen zu beobachten.

Die Sättel und Mulden sind teils offen, teils annähernd isoklinal; größere Falten weisen meist runde, kleinere bevorzugt spitze Scharniere auf. Ein besonderes Merkmal des geologischen Baues sind der Wechsel weitspannig gefalteter Bereiche (W-Abschnitt) und Zonen intensiver Klein-Fältelung (Mittel-Abschnitt) sowie die knickbandartige Anordnung einzelner Strukturen. Diese Knickbänder sind z.T. als Vorstufen in der Entwicklung von Aufschiebungen anzusehen (EAbschnitt). Die Verwerfungen fallen überwiegend steil in nordöstliche Richtungen ein; für manche Störungen läßt sich anhand von Leithorizonten ein Aufschiebungscharakter nachweisen. Danben kommen andere, z.T. entgegengesetzt einfallende Scherflächen vor, die wie Abschiebungen aussehen; sie lassen sich als rotierte ältere Aufschiebungen deuten. Nur wenige Störungen sind wirklich als »Flächen « zu bezeichnen. Meist bilden sie Scherzonen (Ruscheln) von wenigen Millimetern bis zu mehreren Dezimetern Breite (anscheinend mit dem Versatzbetrag steigend).

Die Trennflächen gruppieren sich in die aus der Faltentektonik bekannten Kategorien. Neben steilstehenden Querklüften (ac), Längsklüften (bc) und Lagerklüften ( $a b$, sehr untergeordnet) treten mehrere Systeme von Diagonalklüften auf, unter denen SE- und NW-einfallende Okl-Flächenscharen gegenüber hkl-Flächen vorherrschen. Die Verzerrung insbesondere der acFlächen-Maxima (Diagram in Abb. 3) spiegelt die Streuung der Faltenachsenlagen wider. Aus der Westvergenz des Faltenbaues geht hervor, $\mathrm{da} \beta$ die Stauchung durch einen Gletscher mit westlicher Fließrichtung erzeugt wurde. Dabei ist nach neueren Kartierungen (Menke 1982) das gesamte in Abbau stehende Tonvorkommen als große Schuppe aus dem Untergrund gelöst und vermutlich über mehrere Kilometer verfrachtet worden. Die Basis der Stauchmoräne liegt zwischen etwa $-25 \mathrm{~mm} \mathrm{NN}$ und $-100 \mathrm{~m} \mathrm{NN}$ (Gelän- dehöhe ca. +40 m NN) (Menke 1982: 422-423). Da sichere Hinweise auf mehrmalige Deformation durch verschiedene Saale-Gletscher bisher nicht vorliegen, sind die Stauchungen möglicherweise während einer einzigen Gletscherüberfahrung gebildet worden. Die Ungleichartigkeit des geologischen Baues und die Streuung der Faltenachsenraumlagen sind wahrscheinlich z.T. auf grundbruchähnliche subglaziale Schollenrotationen zurückführen, eine Folge zeitlicher und räumlicher Änderungen von statischer und dynamischer Belastung sowie Fließrichtung und geschwindigkeit des Eises. Daneben dürften durch plastische Deformation (»Zweite Faltung«) \pm transversale Verbiegungen entstanden sein, die die Achsen der älteren Falten umso stärker auslenkten, je größer deren Vergenz war.

\section{Subglazial-distale Stauchmoränen}

\section{Beispiel Großenaspe}

In der Sandgrube Großenaspe nördlich Hamburg sind gestauchte saalezeitliche Sedimente aufgeschlossen. Sie umfassen glazifluviale kiesige Sande und stark steinige Grobkiese sowie sandige Geschiebemergel und, untergeordnet, glazilakustrine Silte und Tone (Abb. 2).

Der sehr ungleichförmige geologische Bau besteht aus Klein-Schuppen, Falten, Schollen und kombinierten Strukturtypen. Im Bau einzelner Strukturen sind Übergänge von anscheinend ungestörtem sedimentärem Primärgefüge zu ausgeprägter Innendeformation zu beobachten. Flache Schichtlagerung tritt gegenüber Steilstellung und Überkippung weitgehend zurück; sie ist z.T. sekundär (s. unten) und nicht als Hinweis auf ungestörte Lagerung zu werten. Die als solche erkennbaren Schichtflächen fallen bei insgesamt starker Streuung bevorzugt in NE- und SW-, die Scherflächen vor allem in NE-Richtungen ein. Der größere Teil der Achsen streicht ungefähr NW/SE, daneben kommen um bis zu $90^{\circ}$ abweichende Achsenrichtungen vor. Infolge \pm transversaler Wellungen im Meter- bis Dekameterbereich sind die Achsen meist geneigt, wobei wechselseitige Abtauchwinkel bis $\mathrm{zu} 50^{\circ}$ vorkommen. Extreme Faltenformen finden sich u.a. in Gestalt flach ausgewalzter Mulden mit subhorizontalen Achsenflächen; ihre hangenden 


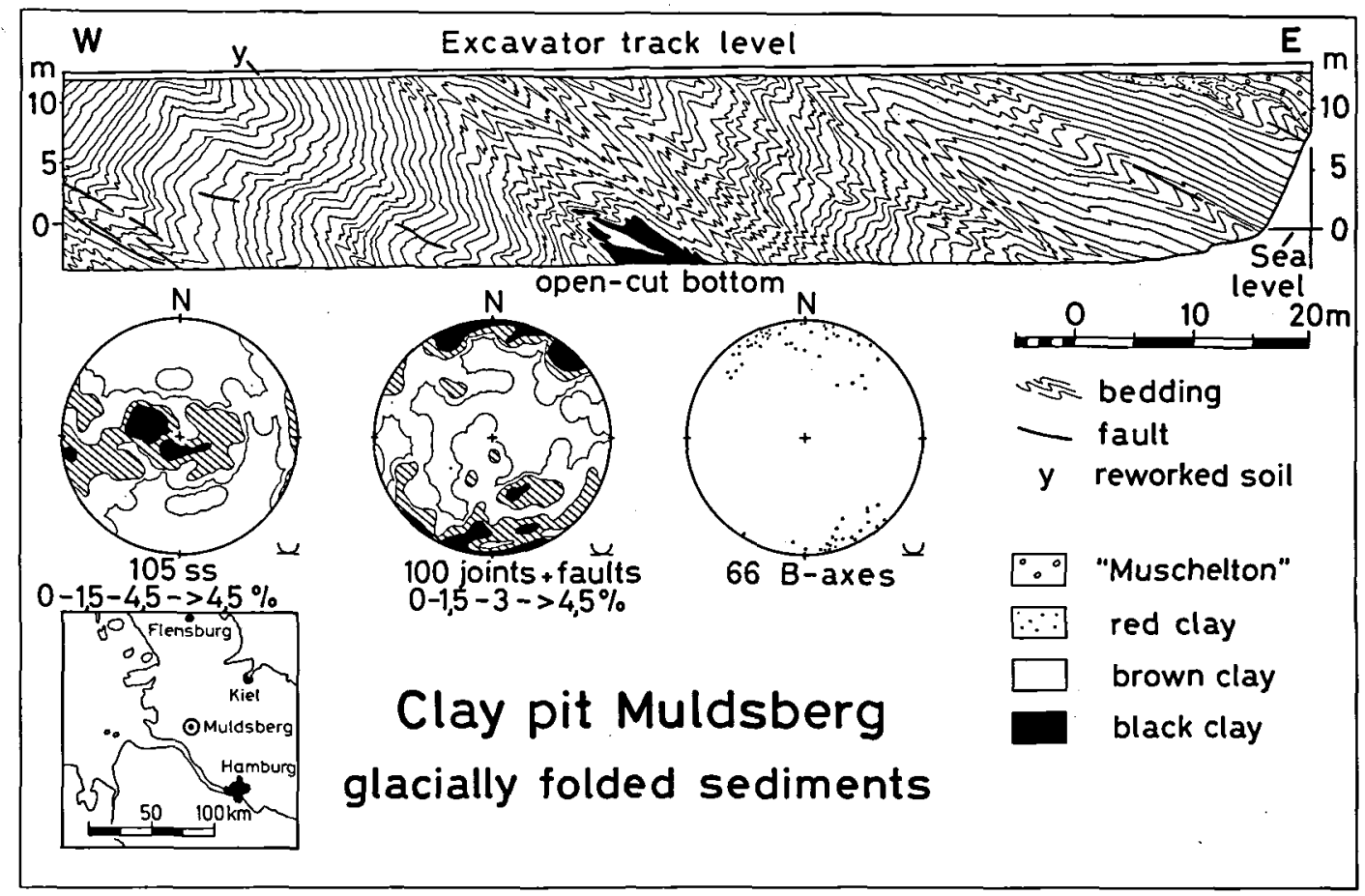

Abb. 3. Saalezeitliche Intensivfältelung elster- und holsteinzeitlicher Meerestone; Tongrube Muldsberg, Ostteil des nördlichen Abbaustoßes, Darstellung als Saigerriß; T. Vollmer, 1982, Originalmaßstab 1:100.

Fig. 3. Intense folding of elsterian and holsteinian marine clay by saalian glacier; clay pit Muldsberg, eastern part of northern slope, shown as vertical section; T. Vollmer, 1982, original scale was 1:100.

Flügel sind um bis $\mathrm{zu}>180^{\circ}$ überkippt (nachweisbar anhand von Schrägschichtung).

Die Schuppenstrukturen umfassen ein breites Spektrum von relativ einfach gebauten Gleitbrettern mit scheinbar geringer Innendeformation (N-Abschnitt) bis zu verwickelten, stark innendeformierten Formen (S-Abschnitt). An manchen Stellen ist zu beobachten, daß mehrere Meter mächtige Partien eines liegenden Geschiebemergels hobelspanartig aufgeschuppt, steilgestellt und in Schubrichtung (ca. SW) zu wenige Zentimeter dünnen Lagen ausgewalzt sind, die distal in schwach siltigen Sand übergehen. Diese ss-parallel in glazifluviale Sande eingeschichteten Lagen sind wenig auffällig, sie können bei ungünstigen Aufschlußverhältnissen mit sedimentären Bildungen verwechselt werden und Konkordanz vortäuschen. Im vorliegenden Aufschluß sind sie ein sicheres Merkmal von Scherflächen oder -zonen. An diesem Beispiel zeigt sich auch, daß bereits nach wenigen Metern tektonischen Transports eine vollständige Mischung verschiedener
Sedimente bis zur weitgehenden Absorption des mengenmäßig untergeordneten Partners eintreten kann.

Manche Scherfläche wirkt auf gewisse Erstrekkung wie eine ungestörte Schichtfläche, die sich einem begrenzten Ausschnitt des Gesteinsverbandes scheinbar konkordant einfügt. Wenige Meter weiter aber (Bedeutung der Aufschlußgröße!) herrscht an derselben Fläche Diskordanz, verbunden mit Schleppung und z.T. meßbarem Versatz von Leithorizonten um mehrere Meter bis ?Zehnermeter. Auch weitgehend im ursprünglichen Verband erhaltene einheitliche Schmelzwassersandkomplexe weisen Anzeichen für interne Verschiebungen entlang von Schichtflächen auf: Die mit ca. $10^{\circ}$ bis $30^{\circ}$ bankschräg einfallenden Leeblätter des primären Schrägschichtungsgefüges versteilen auf wenigen Metern Aufschlußlänge bis zu bankrechter Orientierung; eine Deformation durch subaquatische Gleitung ist auszushließen, da eindeutige Beziehungen zu anderen glazitektonischen Phänome- 
nen bestehen. Die Überprägung des Schrägschichtungsgefüges ist damit eine Folge ungleichförmiger Scherung von räumlich wechselndem Betrag.

Das chaotische Nebeneinander zerscherter Falten und gefalteter Scherflächen erlaubt wohl eine Deutung einzelner Bewegungsabläufe, nicht aber eine genaue Gesamtkonstruktion der Entwicklungsgeschichte dieses Stauchkomplexes. Sicher ist, daß an der Strukturbildung eine Vielzahl teils zusammenwirkender, teils räumlich und zeitlich wechselnder subglazialer Schuppungsund Faltungsvorgänge beteiligt war.

Am Beispiel Großenaspe wird der mögliche Einfluß der Aufschlußgröße auf die genetische Beurteilung von Stauchkomplexen deutlich: Während eines früheren Abbaustadiums wurde ein weit verbreiteter hangender Geschiebemergel zunächst als Grundmoräne des Gletschers angesehen, der die Stauchungen verursacht hat. Erst bei fortgeschrittenem Abbau zeigte sich, daß dieser Geschiebemergel nur örtlich einer flachlagernden Grundmoräne ähnelt, sonst aber in die Stauchung einbezogen ist. Eine echte Deckmoräne wurde bisher nicht gefunden; möglicherweise ist sie abgetragen. Oder gehörte der besagte Geschiebemergel vielleicht doch zur Grundmoräne des stauchenden Gletschers und wurde von diesem in einer letzten Bewegungsphase mit den älteren Schichten verknetet?

\section{Sekundäre Veränderungen des geologischen Baues}

Von durch Gletscherschub und -auflast erzeugten Primärgefügen sind sekundäre Strukturen oder Störungen zu unterscheiden. Sie entstehen durch periglaziale Phänomene wie Eiskeile, Bodeneis, Kryoturbation, Steilstellung von Steinen, fossile Grundbüche und postglaziale Prozesse wie Senkung über Toteis, Verkarstung (z.B. Kalke von Levanger/Drontheim, Scheibkreide auf Mön, in Hemmoor und Lägerdorf) und Subrosion. Abb. 1 zeigt ein Beispiel sekundärer Baustörungen durch Auflösung von permischen Evaporitgesteinen im Untergrund, die zum Einbruch von Erdfällen geführt hat. Die Basis der DrentheGrundmoräne, sonst auf ca. $-5 \mathrm{~m} \mathrm{NN}$ bis $+18 \mathrm{~m}$ NN, ist im Erdfallbereich im nördlichen Profilabschnitt auf mehr als $-20 \mathrm{~m}$ NN abgesenkt wor-

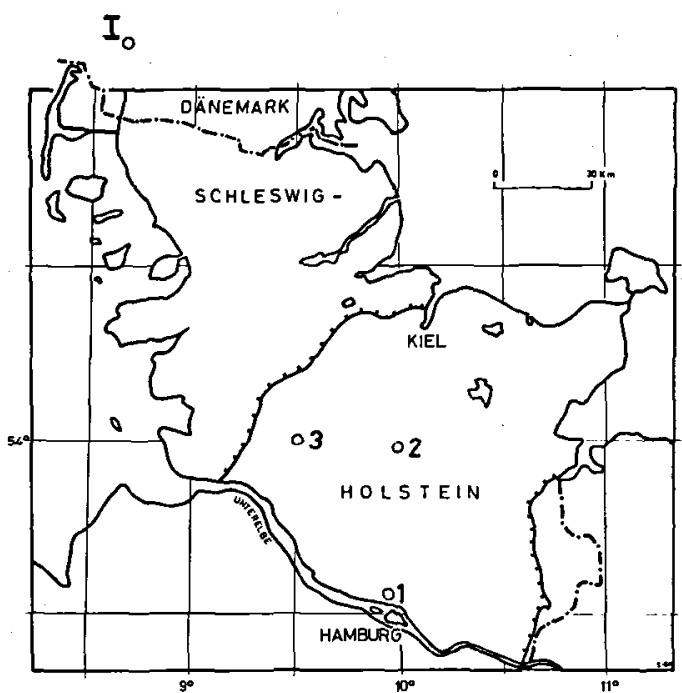

Abb. 4. Lageplan der beschriebenen Aufschlüsse.

Fig. 4. Outcrop map.

den. Die Absenkung muß mindestens eemzeitlich eingesetzt haben. In Einzelfällen können durch subaerisches oder subaquatisches Bodenfließen und Rutschungen (Luhetal, Niedersachsen) Konvergenzformen zu glazitektonischen Strukturen entstehen.

\section{Karten- und Modelldarstellung}

Die exakte Erfassung der komplizierten Glazialtektonik ist trotz sorgfältiger Dokumentation der natürlichen und künstlichen Aufschlüsse schwierig. Daher war die Entwicklung eines Modellversuches mit neuen Werkstoffen (Sand-Paraffingemenge mit eingelagerten Gipsschichten) durch

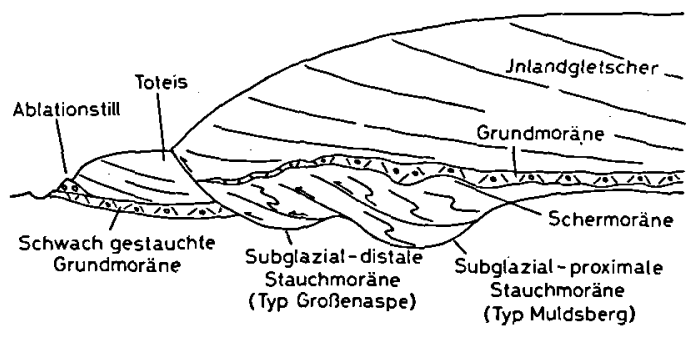

Abb. 5. Schema der Anordnung von Morànentypen im westlichen Holstein, unmaßstäblich, Entwurf T. Vollmer.

Fig. 5. Distribution pattern of different till types in Western Holstein, unscaled, sketch by $\mathrm{T}$. Vollmer. 
Köster (1957) eine wertvolle Bereicherung der Rekonstruktionsverfahren glazitektonischer Vorgänge. Köster gelang es mit dieser Methode nicht nur das Geschehen an der Gletscherstirn, sondern auch subglaziale Vorgänge nachzuahmen.

Durch geologische Kartierung (Berthelsen 1978; Petersen 1978) ist eine kartenmäßige Darstellung der Schichtköpfe steilstehender Stauchschuppen möglich; um deren räumlichen Bau zumindest in seinen Grundzügen zu erfassen, sind beim Fehlen weiterer Aufschlüsse spezielle Strukturkarten auf der Basis tieferer Bohrungen erforderlich. Entsprechend wertete Khoo (1972) ein dichtes Netz tieferer Bohrungen aus, um die räumliche Lage von Tonschollen zu konstruieren. In der Bodenmechanik dienen gelegentliche Modelle aus Plexiglas und Draht zur anschaulichen Darstellung glazial deformierten Untergrundes; Blockbilder sind von Berthelsen (1981) und Wateren (1981) entworfen worden. Fiedler (1975) analysierte glazialtektonische Strukturen mit Hilfe von Blocklackfilmen.

Neben Karten- und statischen Modelldarstellungen, die großenteils den angewandten Geowissenschaften und der geologischen Dokumentation dienen, dürften dynamische Modellversuche und der Vergleich ihrer Ergebnisse mit echten glazitektonischen Phänomenen weiterhin vieles zum genetischen Verständnis der Moränen beitragen.

\section{Dansk sammendrag}

Ud fra en dynamisk betragtning omfatter begrebet moræne både de sedimenter, der er aflejret direkte fra isen, men også de sedimenter, der er blevet transporteret og/eller bevæget $i$ forbindelse med isoverskridelsen. Undergrænsen for denne dynamiske enhed er derfor de, ved det aktuelle isfremstød, ikke deformerede sedimenter.

Morænen ( $=$ dynamisk enhed) opdeles i Nordtyskland normalt i Bundmoræne $(=$ Grundmoräne $=$ till) og Sålmoræene (= Sohlmoräne $=$ »deformation till og $»$ shear till ).

Bundmoræner (till) fra de enkelte isoverskridelser varierer $i$ tykkelse normalt fra få metre til 30 meter, lokalt kendes tykkelser på mere end $80 \mathrm{~m}$. Bundmoræneme fra de enkelte fremstød er ofte homogene inden for større områder m.h.t. petrografisk sammensxtning og viser ofte ensartethed $i$ strukturer (f.eks. forkløftninger) såfremt disse er en primær egenskab opstået som resultat af isoverskridelsen.

Sålmoræner (Shear-, Stauch- og Schmelzmoräne) består generelt af de bevagede og deformerede sedimenter ved isoverskridelsen, og afhængig af underlagets beskaffenhed vil sålmoranerne derfor have vekslende karakter.

Sålmoræner optræder med indtil tre forskellige facies, 1) shear-moræner, der består af udvalset materiale i $\mathrm{mm}$ til $\mathrm{cm}$ tykke horisonter ofte vekslende mellem sand og moræneler, der kan ligne en lagdeling. 2) Smelzmoränen (= "smeltemoræ- ner «), der oftest forekommer umiddelbart under bundmorænen og adskiller sig fra denne ved et hyppigt større sandindhold. Stenpartikler i smeltemorænen viser tit en streng parallellitet med isbevægelsesretningen. 3) Stauchmoränen, der omfatter "Schuppen « og "Schollen«. Schuppen er betegnelsen for korttransporterede og velafgrænsede legemer (flager) adskilt af overskydningsplaner, hvori der næsten aldrig ses nogen egentlig opblanding af materialer. Schollen benyttes om mere diffuse legemer, oftest langtransporterede og præget af det omgivende materiale.

Fra udvalgte lokaliteter i Nordtyskland (se fig. 4) gennemgås eksempler på de forskellige morænetyper, deres karakterer og formodede genese.

\section{Literatur}

Berthelsen, A. (1981): Principles of cold glacial tectonics. INQUA Konferenz Moen.

Berthelsen, O. (1978): Den geologiske kortlægning af Danmark. Danm. geol. Unders $\phi$ gelse, A 2: $73 \mathrm{~S}$.; København.

Boulton, G. (1981): Processes and forms of subglacial deformation. INQUA Konferenz Moen.

Braun, F. J. (1964): Endmorảnen-Stauchwall und Eisrandbildungen bei Moyland/Ndrh. Der Niederrhein, 2: 58-63; Krefeld.

Carlé, W. (1938): Das innere Gefüge der Stauch-Endmoränen und seine Bedeutung für die Gliederung des Altmoränengebietes. Geol. Rundschau, 29 (1/2): 27-51; Stuttgart.

Carlé, W. (1939): Das Messen der inneren Formen in Stauchendmoränen und Sandern. - Aus d. Heimat, 52 (4): 97109; Öhringen.

Carlé, W. (1939a): Die Lagerungsstörungen in den Kieselgurgruben von Ohe (Lüneburger Heide). Abh. Naturw. Ver. Bremen, 31 (2): 221-235; Bremen.

Fiedler, K. (1975): Lackfilme im geologischen Unterricht. Mitt. Geol.-Paläont. Inst. Univ. Hamburg, 44: 331-342; Hamburg.

Grimmel, E. (1969): Ist die nordöstliche Lüneburger Heide eine "Endmoränen «-Landschaft? Eiszeitalter $u$. Gegenwart, 20: 243-244; Öhringen.

Gripp, K. (1964): Erdgeschichte von Schleswig-Holstein. 411 S.; Neumünster (Wachholtz).

Gripp, K. (1975): 100 Jahre Untersuchungen über das Geschehen am Rande des nordeuropäischen Inlandeises. Eiszeitalter $u$. Gegenwart, 26: 31-73; Öhringen.

Grube, F. (1967): Die Gliederung der Saale-(Riss) Kaltzeit im Hamburger Raum. Frühe Menshheit u. Umwelt, 2: 168195; Köln (Böhlau).

Grube, F. (1972): Zur Gliederung der Jungmoränen-Landschaft von Hoisbüttel bei Hamburg. Jb. Alsterver.: $9 \mathrm{~S}$;; Hamburg.

Grube, F. \& Homci, H. (1975): Geologie und Geomorphologie des südlichen Stellmoorer Tunneltales. In: Tromnau, G.: Neue Ausgrab. Ahrensb. Tunneltal: 94-98; Neumünster (Wachholtz).

Houmark-Nielsen, M. (1981): Glacialstratigrafi i Danmark øst for Hovedopholdslinien. - Dansk geol. Foren.: 61-76; København.

Jacobsen, E. M. (1981): Some glaciotectonic features from the southern part of Sealand, Denmark. - INQUA Konferenz Moen.

Khoo, F. (1972): Die Geologie im Bereich des neuen Elbtustnels (Hamburg) und der Tongruben in Wacken (Holstein) sowie die Sedimentpetrographie der Tone der Holstein-Warmzeit. - Unveröff. Dipl.-Arb., Hamburg, 89 S. 
Knudsen, K. L. (1980): Foraminiferal faunas in marine Holsteinian Interglacial deposits of Hamburg-Hummelsbüttel. Mitt. Geol.-Paläont. Inst. Univ. Hamburg, 49: 193214; Hamburg.

Köster, R. (1957): Schuppung und Faltung im glazialtektonischen Experiment. Geol. Rundschau, 46: 564-571; Stuttgart.

Lang, H. D. (1963): Das Endmoränengebiet der Mellendorfer und Brelinger Berge. Ber. Naturhist. Ges., 107: 21-30; Hannover.

Menke, B. (1982): Mittel- und Jungpleisozän in Westholstein am Beispiel des Blattes 1922 Schenefeld. Die Heimat Z. Natur- u. Landeskunde Schleswig-Holst. u. Hmbg., 89 (11/ 12): 419-429; Neumünster.

Mickelson, D. M. \& Waldemarson, D. \& Johansson, H. G (1981): Jointing in till: an example from south-central Sweden. Inqua Commission GLOD, Symposium Wyoming.

Nielsen, P. E. (1981): Till fabric reorientated by subglacial shear. INQUA Konferenz Moen.

Petersen, K. S. (1978): Anwendung glaziotektonischer Untersuchungen bei der geologischen Kartierung in Dänemark. Eiszeitalter u. Gegenwart, 28: 126-132; Öhringen.

Prange, W. (1975): Gefügekundliche Untersuchungen zur Entstehung weichselzeitlicher Ablagerungen an Steilufern der Ostseeküste, Schleswig-Holstein. Meyniana, 27: 41-54 Kiel.

Rappol, M. (1983): Glacigenic Properties of Till, Studies in glacial sedimentology from the Allgäu Alps and The Netherlands. Fys.-Geogr. Univers., 34: 1-225; Amsterdam.

Richter, K. (1929): Studien für fossile Gletscherstruktur. Z Gletscherkde., 17 (1-3): 33-46; Berlin (Bornstraeger).

Richter, K. (1929a): Die Struktur des Warsower Plateaus, ein Beitrag zur Kenntnis der Staumoränen. Abh. Ber. Pommerschen Naturforsch. Ges., 10: 6-12.
Richter, K. (1930): Die Textur des Geschiebemergels und ihre Bedeutung für die Erforschung des präglazialen Untergrundes. Z. Geschiebeforsch., 6 (2): 80-88; Berlin (Borntraeger).

Ruegg, G. H. J. \& Zandstra, J. G. (1981): Geology and archaeology of pleistocene deposits in the ice-pushed ridge near Rhenen and Veenendaal. Med. rijks geol. Dienst. 35: 163268; Haarlem.

Ruszczynska-Szenajch, H. (1981): Coexistence of glacitectonicand glacidynamic structures, examined with a sedimentological approach. Vortrag INQUA Comm. GLQD, Symposium Moen.

Stephan, H. J. (1981): Deformations striking parallel to glacier movement as a problem in reconstructing its direction. INQUA Konferenz Moen.

Stephan, H. J. (1982): Proposal for German terms for some types of till. Unveröff. Vorschlag INQUA Kongreß Moskau.

Valeton, I. \& Löhnert, E. \& Khoo, F. (1972): Genese pleistozäner Rottone im Raume Hamburg. Mitt. Geol.-Paläont. Inst. Univ. Hamburg, 41: 171-184; Hamburg.

Valeton, I. (1980): Zur Petrographie und Geochemie des Holstein-Interglazials von Hamburg-Hummelsbüttel. Mitt. Geol.-Paläont. Inst. Univ. Hamburg, 49: 175-192; Hamburg.

Wateren van der, F. M. (1981): Glacial tectonics near Rhenen (the central Netherlands) and some remarks on the mecha nism that caused them. Vortrag INQUA Comm. GLQD, Symposium Moen.

Wee, M. W. ter (1962): The Saalian glaciation in the Netherlands. Meded. Geol. St., N. S., 15: 57-76; Haarlem.

Wilke, H. (1982): Quartärgeologie des Elbhanges im Westen von Hamburg. Unveröff. Dipl.-Arb., Hamburg, $140 \mathrm{~S}$. 